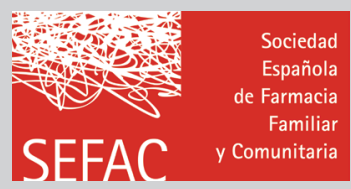

\title{
El farmacéutico comunitario, profesional necesario en la prevención y control de las enfermedades cardiovasculares
}

\section{Rosa Prats}

Vocal de la Junta Directiva de SEFAC. Coordinadora del Grupo de HTA y Riesgo vascular.

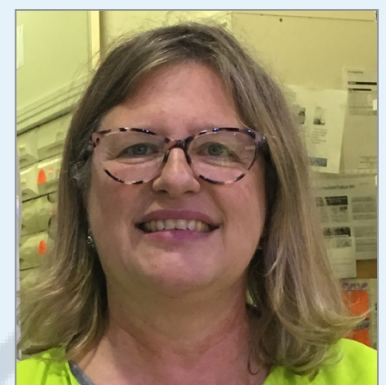

Rosa Prats

\section{PALABRAS CLAVE}

Enfermedades

cardiovasculares (ECV),

farmacéutico comunitario,

hipertensión arterial (HTA),

hipertensión enmascarada,

presión arterial (PA)

\section{KEYWORDS}

Cardiovascular diseases (CVD), community drug, arterial hypertension (AHT), masked hypertension, blood pressure (BP)
Las enfermedades cardiovasculares (ECV) siguen siendo la principal causa de muerte en Europa occidental y en España, representando la hipertensión arterial (HTA) el factor de riego más prevalente de estas patologías. Hablamos también de los accidentes cerebrovasculares, infartos, problemas renales, etc. Según la Organización Mundial de la Salud (OMS) estas enfermedades son, también, la primera causa de muerte en el mundo. Mientras las poblaciones al envejecer adopten un estilo de vida más sedentario y aumenten el peso corporal, la prevalencia de la HTA seguirá creciendo en todo el mundo. Se calcula que el número de personas con HTA aumentará en un 15-20\% en 2025 (1).

Por ello es de gran importancia su detección precoz y la instauración de un tratamiento adecuado que permita alcanzar el objetivo terapéutico. Las causas de la presión arterial (PA) alta dependen en gran medida de factores ambientales identificados como el sobrepeso, la ingesta excesiva de alcohol y sal alimenticia y un nivel insuficiente de actividad física, por lo que con algunos cambios en los hábitos de vida podrían prevenirse. No obstante, la detección y tratamiento resultan muchas veces complicados debido a varios motivos: es asintomática, hay poca conciencia de enfermedad por parte del paciente $\mathrm{y}$, en los pacientes ya diagnosticados $\mathrm{y}$ tratados, frecuentemente el control no es el óptimo debido al incumplimiento terapéutico. Por todo ello, es importante conocer distintos aspectos epidemiológicos de la HTA en nuestro país, que pueden ir desde su prevalencia, al grado de control y los tratamientos utilizados.

La Encuesta Nacional de Salud 2017 del Ministerio de Sanidad (2) establece que el porcentaje de hipertensos diagnosticados en España es de un 19,8 \%, siendo la primera enfermedad o proble- ma de salud en la población, con un $13 \%$ para el tramo de edad de 25 a 64 años y del 51,9\% en el de 65 o más años. Esta prevalencia va en aumento, ya que en el período 1993-2017 ha pasado de 11,2 \% a 19,8\%, aumentado también otros factores de riesgo de ECV como el colesterol y la diabetes, con las consecuencias que ello supone.

El control de la presión arterial mediante la toma de la presión arterial es un procedimiento sencillo, rápido y no invasivo, que permite detectar cifras de presión arterial elevadas con el fin de iniciar las medidas necesarias para prevenir las complicaciones cardiovasculares. En general, esta medición es accesible a toda la población, ya que la farmacia comunitaria presta este servicio de manera protocolizada y, a veces, el farmacéutico es el único profesional sanitario disponible o el primero consultado ante un problema de salud, por lo que su participación en cribados poblacionales, control y seguimiento de estos pacientes es muy importante.

Para la formación y capacitación del farmacéutico comunitario en el abordaje de la PA y del RV, existe el programa impacHta, promovido por la Sociedad Española de Farmacia Familiar y Comunitaria (SEFAC) y la Sociedad Española de Hipertensión Arterial (SEH-Lehla), en el cual hay cerca de 2.000 matriculados repartidos por todas las comunidades autónomas. Este programa les facilita el registro del seguimiento y control del paciente en la plataforma SEFAC e_XPERT (www.sefacexpert.org), donde además se puede realizar una revisión de la medicación, gestionar agenda de citas, informes de derivación e información al paciente.

Diez millones de personas pierden la vida cada año en el mundo debido a una PA alta y solo la mitad de los hipertensos conocen su estado, es por ello 
que es necesario realizar cribados poblacionales. Por esta razón las farmacias españolas se han unido un año más a la campaña internacional anual MMM (May Measurement Month), para medir la presión arterial durante el pasado mes de mayo. El objetivo de esta campaña, que ya lleva varias ediciones, es sensibilizar a la población mundial de la importancia de conocer sus cifras de presión arterial (PA) y aportar información sobre hábitos de vida saludable y los tratamientos más adecuados en aquellas personas con hipertensión arterial. La campaña MMM está promovida por la Sociedad Internacional de Hipertensión y la Liga Mundial contra la Hipertensión Arterial y en España colaboran distintas sociedades científicas, siendo liderada en el ámbito farmacéutico por SEFAC.

Una de las novedades de la última edición en España (MMM19) es que la campaña sirve de base para la realización entre SEFAC y SEH-Lelha del estudio HENFAC para detectar la denominada 'hipertensión enmascarada', utilizando la automedida de la presión arterial (AMPA) para su determinación. La HTA enmascarada se asocia con un aumento de la morbimortalidad cardiovascular. Se debe sospechar cuando se observen discrepancias entre las cifras domiciliarias y clínicas.

Por otro lado, sabemos que la HTA es uno de los factores de riesgo más importantes para desarrollar la fibrilación auricular (FA), que es la arritmia más importante como causa de accidente cerebrovascular (ACV), insuficiencia cardiaca (IC), muerte súbita y morbilidad cardiovascular (CV) a nivel mundial, y con una tendencia significativa a aumentar (3). Por este motivo SEFAC también colabora con Atrial Fibrillation Asosociation (AFA) y la Internacional Pharmacists Anti Coagulacion Taskforce (IPACT) en el proyecto internacional para valorar su eficacia en la mejora de la detección de fibrilación auricular (FA) asintomática, la reducción de sus consecuencias y contribuir al correcto tratamiento.

\section{Resultados destacables}

La importancia de estos cribados poblacionales en farmacia comunitaria los podemos ver en los resultados de los MMM de los últimos años y de las campañas del proyecto Know your pulse. Durante el proyecto MMM17 se realizaron 3.849 mediciones en España, correspondiendo 3.267 a los datos de las farmacias comunitarias españolas, poniendo en valor la alta participación del farmacéutico. Es importante el hecho de que el $42,7 \%$ de los pacientes no sabían el tiempo que hacía que se habían tomado la presión. Los resultados de cribado de la PA en España revelaron la existencia de un elevado número de personas con la PA elevada entre la población adulta (25,6\%), de los que más del $40 \%$ no recibian tratamiento antihipertensivo. El nivel de control de la PA entre los hipertensos en tratamiento no es óptimo en la muestra analizada y es todavía peor entre los diabéticos hipertensos, lo que indica un deficiente seguimiento o una inercia terapéutica contraria a las recomendaciones de las guías clínicas actuales. En MMM18 se hicieron en las farmacias comunitarias españolas 5.785 mediciones, detectando un 31,18\% de los valores PAS y/o PAD $\geq 140 / 90 \mathrm{mmHg}$. Un tercio de los encuestados hacía más de un año que se había medido la PA. En Know your pulse se pudo concluir la importancia de detectar la existencia de pacientes con PA no controlada y pulso irregular para prevenir los eventos relacionados.

Debido a que la HTA continúa siendo la mayor causa evitable de enfermedad cardiovascular y de mortalidad por cualquier causa tanto en Europa como en el mundo (4-5) los profesionales sanitarios debemos de aunar esfuerzos en pro de prevenirla, reforzar el control y seguimiento del paciente. Es aquí donde tiene un papel importante el farmacéutico comunitario, ya que es un profesional cercano y formado que puede aportar información y contribuir a mejorar el grado de control de la HTA y con ello de las ECV.

\section{Referencias bibliográficas}

1. NCD Risk Factor Collaboration. Worldwide trends in blood pressure from 1975 to 2015: a pooled analysis of 1479 population-based measurement studies with 19.1 million participants. Lancet. 2017;389:37-55.

2. ENSE. Encuesta Nacional de Salud España 2017 [Monografia en Internet] Madrid. Ministerio de Sanidad, Consumo y Bienestar Social. 2017. Disponible en http://www.infocoponline.es/ pdf/ENSE17.pdf

3. Grupo de Trabajo de la Sociedad Europea de Cardiología (ESC) para el diagnóstico y tratamiento de la fibrilación auricular. Guía ESC 2016 sobre el diagnóstico y tratamiento de la fibrilación auricular, desarrollada en colaboración con la EACTS. Rev Esp Cardiol. 2017;70. doi:10.1016/j.recesp.2017.07.028

4. Banegas JR, Lopez-Garcia E, Dallongeville J, Guallar E, Halcox JP, Borghi C, Masso- Gonzalez EL, Jimenez FJ, Perk J, Steg PG, De Backer G, Rodriguez-Artalejo F. Achievement of treatment goals for primary prevention of cardiovascular disease in clinical practice across Europe: the EURIKA study. Eur Heart J. 2011;32:2143-2152. doi:10.1093/eurheartj/ehr080

5. Chow CK, Teo KK, Rangarajan S, Islam S, Gupta R, Avezum A, Bahonar A, Chifamba J, Dagenais G, Diaz R, Kazmi K, Lanas F, Wei L, Lopez-Jaramillo P, Fanghong L, Ismail NH, Puoane T, Rosengren A, Szuba A, Temizhan A, Wielgosz A, Yusuf R, Yusufali A, McKee M, Liu L, Mony P, Yusuf S, PURE Study Investigators. Prevalence, awareness, treatment, and control of hypertension in rural and urban communities in high-, middle-, and low-income countries. JAMA. 2013;310:959-968. doi:10.1001/jama.2013.184182 